\title{
SURFACES WITH POINTWISE 1-TYPE GAUSS MAP
}

\author{
Dong-SoO KIM
}

\begin{abstract}
In this article, we study generalized slant cylindrical surfaces (GSCS's) with pointwise 1-type Gauss map of the first and second kinds. Our main results state that GSCS's with pointwise 1-type Gauss map of the first kind coincide with surfaces of revolution with constant mean curvature; and the right cones are the only polynomial kind GSCS's with pointwise 1-type Gauss map of the second kind.
\end{abstract}

\section{Introduction AND PRELIMINARIES}

The notion of finite type submanifolds in Euclidean or pseudo-Euclidean space, introduced by B.-Y. Chen during the late 1970's, has become a useful tool for investigating and characterizing many important submanifolds (cf. [3, 4]). In [1, 2, 6] the notion of finite type was extended to differential maps, in particular, to Gauss map of submanifolds.

If a submanifold $M$ of Euclidean or pseudo-Euclidean space has 1-type Gauss map $G$, then $G$ satisfies $\Delta G=\lambda(G+C)$ for some $\lambda \in \mathbb{R}$ and some constant vector $C$, where $\Delta$ is the Laplace operator corresponding to the induced metric on $M$ (cf $[1,2,9])$. However, the Laplacian of the Gauss map of several important surfaces such as helicoids, catenoids and right cones take a somewhat different form; namely,

$$
\Delta G=f(G+C)
$$

for some non-constant function $f$ and some constant vector $C$. For this reason, a submanifold is said to have pointwise 1-type Gauss map if its Gauss map satisfies (1.1) for some smooth function $f$ on $M$ and vector $C$. A submanifold with pointwise 1-type Gauss map is said to be of the first kind if the vector $C$ in (1.1) is the zero

Received by the editors September 26, 2011. Revised Oct. 19, 2011. Accepted Oct. 20, 2011. 2000 Mathematics Subject Classification. 53B25, 53C40.

Key words and phrases. cylindrical surfaces, slant cylindrical surfaces, generalized slant cylindrical surfaces, pointwise 1-type Gauss map.

This study was financially supported by Chonnam National University, 2010. 
vector. Otherwise, the pointwise 1-type Gauss map is said to be of the second kind ([5]).

Let $M$ be a surface of Euclidean 3-space $\mathbb{E}^{3}$. The map $G: M \rightarrow S^{2} \subset \mathbb{E}^{3}$ which sends each point of $M$ to the unit normal vector to $M$ at the point is called the Gauss map of the surface $M$, where $S^{2}$ is the unit sphere in $\mathbb{E}^{3}$ centered at the origin.

For the matrix $g=\left(g_{i j}\right)$ consisting of the components of the metric on $M$, we denote by $g^{-1}=\left(g^{i j}\right)$ (resp. $\mathcal{G}$ ) the inverse matrix (resp. the determinant) of the matrix $\left(g_{i j}\right)$. The Laplacian $\Delta$ on $M$ is, in turn, given by

$$
\Delta=-\frac{1}{\sqrt{\mathcal{G}}} \sum_{i, j} \frac{\partial}{\partial x^{i}}\left(\sqrt{\mathcal{G}} g^{i j} \frac{\partial}{\partial x^{j}}\right) .
$$

Here, we give an example of surfaces of revolution with pointwise 1-type Gauss map of the second kind.

Example 1.1. Consider the right cone $C_{a}$ which is parameterized by

$$
x(u, v)=(v \cos u, v \sin u, a v), \quad a \geq 0 .
$$

Then the Gauss map $G$ and its Laplacian $\Delta G$ are respectively given by

$$
G=\frac{1}{\sqrt{1+a^{2}}}(a \cos u, a \sin u,-1)
$$

and

$$
\Delta G=\frac{1}{v^{2}}\left(G+\left(0,0, \frac{1}{\sqrt{1+a^{2}}}\right)\right) .
$$

It implies that the right cone has pointwise 1-type Gauss map of the second kind.

In [5], B.-Y. Chen, M. Choi and Y.H. Kim studied surfaces of revolution with pointwise 1-type Gauss map. In [7], U. Dursun studied flat surfaces in Euclidean 3-space with pointwise 1-type Gauss map.

The author and Y.H. Kim introduced the class of generalized slant cylindrical surfaces (GSCS's) in [8]. This class includes surfaces of revolution and cylindrical surfaces as special cases. Thus, we need to consider the GSCS's in $\mathbb{E}^{3}$ with pointwise 1-type Gauss map.

In this paper, we study the GSCS's with pointwise 1-type Gauss map. In particular, we prove that GSCS's with pointwise 1-type Gauss map of the first kind coincide with surfaces of revolution with constant mean curvature; and the right cones are the only polynomial kind GSCS's with pointwise 1-type Gauss map of the second kind. 
Hereafter, all objects are assumed to be connected and smooth unless mentioned otherwise.

\section{Generalized Slant Cylindrical Surfaces}

For a fixed unit speed plane curve $X(s)=(x(s), y(s), 0)$, let $T(s)=X^{\prime}(s)$ and $N(s)=\left(-y^{\prime}(s), x^{\prime}(s), 0\right)$ denote the unit tangent and principal normal vector, respectively. The curvature $\kappa(s)$ of $X(s)$ is defined by $T^{\prime}(s)=\kappa(s) N(s)$ and we have $T(s) \times N(s)=V$, where $V$ denotes the unit vector $(0,0,1)$. For a constant $\theta$, we let $Y_{\theta}(s)=\cos \theta N(s)+\sin \theta V$. Then the ruled surface $M$ defined by

$$
F(s, t)=X(s)+t Y_{\theta}(s)
$$

is regular at $(s, t)$ where $1-\cos \theta \kappa(s) t$ does not vanish. This ruled surface $M$ is called a slant cylindrical surface $(S C S)$ over $X(s)$. For the unit normal vector $G=-\sin \theta N(s)+\cos \theta V, M$ satisfies

$$
\left\langle F_{s}, F_{t}\right\rangle=0,\left\langle F_{s t}, G\right\rangle=0 .
$$

This shows that the coordinate lines of $F$ are lines of curvature of $M$ with corresponding principal curvatures

$$
k_{1}(s, t)=\frac{-\kappa(s) \sin \theta}{1-\kappa(s) t \cos \theta}, k_{2}(s, t)=0,
$$

respectively. The SCS with $\sin \theta=0$ or $\cos \theta=0$ is nothing but a parametrization of either a plane or a cylindrical surface.

In general, we consider another unit speed plane curve $W(t)=(z(t), w(t))$. If we let $Y_{s}(t)=z(t) N(s)+w(t) V$, then the parametrized surface defined by

$$
H(s, t)=X(s)+Y_{s}(t)
$$

is regular at $(s, t)$ where $1-\kappa(s) z(t)$ does not vanish. This parametrized surface $M$ is called a generalized slant cylindrical surface $(G S C S)$ over $X(s)$. For the unit normal vector $G(s, t)=-w^{\prime}(t) N(s)+z^{\prime}(t) V, M$ satisfies

$$
\left\langle H_{s}, H_{t}\right\rangle=0,\left\langle H_{s t}, G\right\rangle=0 \text {. }
$$

This shows that $H(s, t)$ is a principal curvature coordinate system of $M$ with corresponding principal curvatures

$$
k_{1}(s, t)=\frac{-\kappa(s) w^{\prime}(t)}{1-\kappa(s) z(t)}, k_{2}(s, t)=\kappa(t),
$$

respectively, where $\kappa(t)=z^{\prime}(t) w^{\prime \prime}(t)-z^{\prime \prime}(t) w^{\prime}(t)$ denotes the curvature of $W(t)$. 
If $W(t)$ is a straight line, then the GSCS $H(s, t)$ is nothing but a SCS. If $X(s)$ is a straight line, then the GSCS $H(s, t)$ is nothing but a cylindrical surface. Furthermore, we have the following $([8])$.

Proposition 2.1. If $X(s)$ is a circle, then GSCS $M$ over $X(s)$ is a surface of revolution.

Therefore cylindrical surfaces and surfaces of revolution are special cases of GSCS's.

Now we give the following:

Proposition 2.2. Let $M$ denote a GSCS given by (2.3). Then we have the following.

(1) If the mean curvature $H$ is constant, then $M$ is a surface of revolution.

(2) If the Gaussian curvature $K$ is constant, then $M$ is either a surface of revolution or an SCS.

Proof. It follows from (2.4) that

$$
2 H=\kappa(t)+\frac{-\kappa(s) w^{\prime}(t)}{1-\kappa(s) z(t)}, \quad K=\frac{-\kappa(s) \kappa(t) w^{\prime}(t)}{1-\kappa(s) z(t)} .
$$

Hence we have

$$
\kappa(t)-2 H=\kappa(s)\left\{\kappa(t) z(t)-2 H z(t)+w^{\prime}(t)\right\},
$$

and

$$
K=\kappa(s)\left\{K z(t)-\kappa(t) w^{\prime}(t)\right\}
$$

Suppose that $H$ is constant. If $\kappa(t)-2 H \neq 0$, then (2.6) shows that $\kappa(s)$ is a nonzero constant, and hence $M$ is a surface of revolution. If $\kappa(t)-2 H=0$, then (2.5) implies $\kappa(s) w^{\prime}(t)=0$. In case $\kappa\left(s_{0}\right) \neq 0$ for some $s_{0}, w^{\prime}(t)$ vanishes identically, and hence $M$ is a part of a plane. Otherwise, $\kappa(s)$ vanishes identically. Hence $X(s)$ is a straight line. Thus $M$ is a part of a plane $(H=0)$ or a circular cylinder $(H \neq 0)$.

Now suppose that $K$ is constant. If $K \neq 0$, it follows from $(2.7)$ that $\kappa(s)$ is a nonzero constant, and hence $M$ is a surface of revolution. In case $K=0$ and $\kappa\left(s_{0}\right) \neq 0,(2.7)$ shows that $\kappa(t)$ vanishes identically, and hence $M$ is an SCS. In case $K=0$ and $\kappa(s)$ vanish identically, then $M$ is a cylindrical surface. 


\section{GSCS's with Pointwise 1-Type Gauss Map of the First Kind}

Let $X(s)=(x(s), y(s), 0)$ be a unit speed plane curve with the Frenet frame $\{T(s), N(s)\}$. We consider GSCS's parametrized by

$$
H(s, t)=X(s)+Y_{s}(t),
$$

where $W(t)=(z(t), w(t))$ is a unit speed plane curve, $Y_{s}(t)=z(t) N(s)+w(t) V$, and $V=(0,0,1)$. Then $H(s, t)$ is regular at $(s, t)$ where $Q(s, t)=1-\kappa(s) z(t)$ does not vanish and we get

$$
\begin{aligned}
& H_{s}=Q(s, t) T(s), \quad H_{t}=z^{\prime}(t) N(s)+w^{\prime}(t) V, \\
& G(s, t)=-w^{\prime}(t) N(s)+z^{\prime}(t) V .
\end{aligned}
$$

The Laplacian $\Delta$ on $M$ is given by

$$
\Delta f=-Q^{-3}\left\{\kappa^{\prime}(s) z(t) f_{s}+Q f_{s s}-Q^{2} \kappa(s) z^{\prime}(t) f_{t}+Q^{3} f_{t t}\right\} .
$$

Hence it follows from (3.2) and (3.3) that

$$
\begin{aligned}
-Q^{3} \Delta G= & \kappa^{\prime}(s) w^{\prime}(t) T(s)+Q\left\{\kappa(s)^{2} w^{\prime}(t)+Q \kappa(s) z^{\prime}(t) w^{\prime \prime}(t)\right. \\
& \left.-Q^{2} w^{\prime \prime \prime}(t)\right\} N(s)+Q^{2}\left\{-\kappa(s) z^{\prime}(t) z^{\prime \prime}(t)+Q z^{\prime \prime \prime}(t)\right\} V .
\end{aligned}
$$

Now suppose that $M$ has the pointwise 1-type Gauss map $G$ which satisfies (1.1). Then, letting $C=C_{1}(s) T(s)+C_{2}(s) N(s)+C_{3} V$, we have the following.

$$
\begin{gathered}
\kappa^{\prime}(s) w^{\prime}(t)=-Q^{3} C_{1}(s) f(s, t), \\
\kappa(s)^{2} w^{\prime}(t)+Q \kappa(s) z^{\prime}(t) w^{\prime \prime}(t)-Q^{2} w^{\prime \prime \prime}(t)=Q^{2} f(s, t)\left\{w^{\prime}(t)-C_{2}(s)\right\},
\end{gathered}
$$

and

$$
\kappa(s) z^{\prime}(t) z^{\prime \prime}(t)-Q z^{\prime \prime \prime}(t)=Q f(s, t)\left\{z^{\prime}(t)+C_{3}\right\} .
$$

Using above, we get the following:

Theorem 3.1. Let $M$ be a GSCS given by (3.1). Suppose that $M$ has pointwise 1-type Gauss map $G$ of the first kind. Then $M$ is a surface of revolution.

Proof. Since $C=C_{1}(s) T(s)+C_{2}(s) N(s)+C_{3} V=0$, it follows from (3.5) that $\kappa^{\prime}(s) w^{\prime}(t)=0$. In case $\kappa^{\prime}\left(s_{0}\right) \neq 0$ for some $s_{0}, w(t)$ is constant, and hence $M$ is a part of a plane. Otherwise, $\kappa$ is constant. If $\kappa$ is nonzero, then $M$ is a surface of revolution. If $\kappa=0$, then it follows from (3.6) and (3.7) that

$$
z^{\prime \prime \prime}(t)+f(s, t) z^{\prime}(t)=0, \quad w^{\prime \prime \prime}(t)+f(s, t) w^{\prime}(t)=0 .
$$

This shows that $\kappa^{\prime}(t)=0$. Thus $M$ is a plane or a circular cylinder. 
Combining Theorem 3.1 in [5] and Proposition 2.2, Theorem 3.1 shows directly the following.

Corollary 3.2. Let $M$ be a GSCS given by (3.1). Then the following are equivalent.

(1) $M$ has pointwise 1-type Gauss map $G$ of the first kind.

(2) $M$ has constant mean curvature.

(3) $M$ is a surface of revolution with constant mean curvature.

Remark 3.3. Surfaces of revolution with constant mean curvature are also known as surfaces of Delaunay (cf. [10, p.115]).

\section{GSCS's with Pointwise 1-type Gauss Map of the Second Kind}

Consider a GSCS $M$ parametrized by (3.1). If $M$ is not cylindrical, then $W(t)$ can be parametrized by $W(t)=(t, g(t))$ for some function $g=g(t)$. Hence $M$ is given by

$$
H(s, t)=X(s)+t N(s)+g(t) V .
$$

If $g(t)$ is a polynomial in $t$, Then $M$ is said to be of polynomial kind ([5]). $H(s, t)$ is regular at $(s, t)$ where $Q(s, t)=1-t \kappa(s) \neq 0$ and we get

$$
\begin{aligned}
& H_{s}=Q(s, t) T(s), H_{t}=N(s)+g^{\prime}(t) V, \\
& G(s, t)=\frac{1}{P(t)}\left\{-g^{\prime}(t) N(s)+V\right\}, P(t)=\sqrt{1+g^{\prime}(t)^{2}} .
\end{aligned}
$$

The Laplacian $\Delta$ on $M$ is given by

$$
\begin{aligned}
\Delta f= & -P^{-4} Q^{-3}\left\{\kappa^{\prime}(s) t P^{4} f_{s}+P^{4} Q f_{s s}\right. \\
& \left.-\left(P^{2} Q^{2} \kappa(s)+Q^{3} g^{\prime} g^{\prime \prime}\right) f_{t}+P^{2} Q^{3} f_{t t}\right\} .
\end{aligned}
$$

Hence it follows from (4.2) and (4.3) that

$$
\begin{aligned}
\Delta G= & -\kappa^{\prime}(s) g^{\prime} P^{-1} Q^{-3} T(s) \\
& -P^{-7} Q^{-2}\left\{\kappa(s)^{2} g^{\prime} P^{6}+\kappa(s) g^{\prime \prime} P^{2} Q\right. \\
& \left.+g^{\prime}\left(g^{\prime \prime}\right)^{2} Q^{2}-g^{\prime \prime \prime} P^{2} Q^{2}+3 g^{\prime}\left(g^{\prime \prime}\right)^{2} Q^{2}\right\} N(s) \\
& -P^{-7} Q^{-1}\left\{\left(3\left(g^{\prime}\right)^{2}\left(g^{\prime \prime}\right)^{2}-\left(g^{\prime \prime}\right)^{2}-g^{\prime} g^{\prime \prime \prime}-\left(g^{\prime}\right)^{3} g^{\prime \prime \prime}\right) Q+\kappa(s) g^{\prime} g^{\prime \prime} P^{2}\right\} V .
\end{aligned}
$$

Suppose that the Gauss map $G$ satisfies (1.1) with nonzero constant vector $C$. Then, letting $C=C_{1}(s) T(s)+C_{2}(s) N(s)+C_{3} V$, we have the following.

$$
P Q^{3} C_{1}(s) f(s, t)+\kappa^{\prime}(s) g^{\prime}(t)=0,
$$




$$
\begin{aligned}
& P^{6} Q^{2} f(s, t)\left\{-g^{\prime}(t)+P C_{2}(s)\right\}+\kappa(s)^{2} g^{\prime} P^{6} \\
& +\kappa(s) g^{\prime \prime} P^{2} Q+g^{\prime}\left(g^{\prime \prime}\right)^{2} Q^{2}-g^{\prime \prime \prime} P^{2} Q^{2}+3 g^{\prime}\left(g^{\prime \prime}\right)^{2} Q^{2}=0,
\end{aligned}
$$

and

$$
\begin{aligned}
& P^{6} Q f(s, t)\left\{1+C_{3} P\right\}+\left\{3\left(g^{\prime}\right)^{2}\left(g^{\prime \prime}\right)^{2}\right. \\
& \left.-\left(g^{\prime \prime}\right)^{2}-g^{\prime} g^{\prime \prime \prime}-\left(g^{\prime}\right)^{3} g^{\prime \prime \prime}\right\} Q+\kappa(s) g^{\prime} g^{\prime \prime} P^{2}=0 .
\end{aligned}
$$

It follows from (4.5) and (4.7) that

$$
\begin{aligned}
& C_{3} \kappa^{\prime}(s) g^{\prime} P^{6}+\kappa^{\prime}(s) g^{\prime} P^{5} \\
& =C_{1}(s) Q^{3}\left\{3\left(g^{\prime}\right)^{2}\left(g^{\prime \prime}\right)^{2}-\left(g^{\prime}\right)^{3} g^{\prime \prime \prime}\right\}+C_{1}(s) \kappa(s) g^{\prime} g^{\prime \prime} P^{2} Q^{2} \\
& \quad-C_{1}(s) Q^{3}\left\{\left(g^{\prime \prime}\right)^{2}+g^{\prime} g^{\prime \prime \prime}\right\}
\end{aligned}
$$

Suppose that $M$ is a GSCS of polynomial kind, that is, $g(t)$ is a polynomial in $t$. Denote by $\operatorname{deg} g(t)$ the degree of $g(t)$.

If $\operatorname{deg} g(t)=n \geq 2$, then $P^{2}$ is a polynomial of degree $2 n-2$. By comparing the degree of both sides of (4.8), we see that $C_{3} \kappa^{\prime}(s)=0$, and hence we get

$$
\begin{aligned}
\kappa^{\prime}(s) g^{\prime} P^{5}= & C_{1}(s) Q^{3}\left\{3\left(g^{\prime}\right)^{2}\left(g^{\prime \prime}\right)^{2}-\left(g^{\prime}\right)^{3} g^{\prime \prime \prime}\right\}+C_{1}(s) \kappa(s) g^{\prime} g^{\prime \prime} P^{2} Q^{2} \\
& -C_{1}(s) Q^{3}\left\{\left(g^{\prime \prime}\right)^{2}+g^{\prime} g^{\prime \prime \prime}\right\} .
\end{aligned}
$$

By comparing the degree of both sides of (4.9), we see that $\kappa^{\prime}(s)=0$. Thus, if $\kappa \neq 0$, $M$ is a surface of revolution. If $\kappa=0$, then $T, N$ are constant vectors and $M$ is a cylindrical surface over a plane curve $W(t)$. Since $Q=1$, we have from (4.4)

$$
\begin{aligned}
\Delta G= & -P^{-7}\left\{g^{\prime}\left(g^{\prime \prime}\right)^{2}-g^{\prime \prime \prime} P^{2}+3 g^{\prime}\left(g^{\prime \prime}\right)^{2}\right\} N \\
& -P^{-7}\left\{3\left(g^{\prime}\right)^{2}\left(g^{\prime \prime}\right)^{2}-\left(g^{\prime \prime}\right)^{2}-g^{\prime} g^{\prime \prime \prime}-\left(g^{\prime}\right)^{3} g^{\prime \prime \prime}+\right\} V .
\end{aligned}
$$

Using (1.1), we get $C_{1}=0, C_{2}^{\prime}=C_{3}^{\prime}=0$, and

$$
\left\{1+\left(g^{\prime}\right)^{2}\right\}\left\{C_{2} A-C_{2} B-C_{3} D\right\}^{2}=\left\{g^{\prime} A-g^{\prime} B+D\right\}^{2},
$$

where

$$
\begin{aligned}
& A=3\left(g^{\prime}\right)^{2}\left(g^{\prime \prime}\right)^{2}-\left(g^{\prime}\right)^{3} g^{\prime \prime \prime}, \quad B=\left(g^{\prime \prime}\right)^{2}+g^{\prime} g^{\prime \prime \prime}, \\
& D=4 g^{\prime}\left(g^{\prime \prime}\right)^{2}-g^{\prime \prime \prime}-\left(g^{\prime}\right)^{2} g^{\prime \prime \prime} .
\end{aligned}
$$

By comparing the coefficient of highest degree of both sides of (4.11), we get $C_{2}^{2}=1$, and hence again we get $C_{3}=0$. This shows that the coefficient of highest degree of $g^{\prime} A D$ becomes zero, which is a contradiction.

If $\operatorname{deg} g(t)=1$, then $M$ is a slant cylindrical (non-cylindrical) surface. Note that $P=\sqrt{1+a^{2}}$, where $g^{\prime}(t)=a \neq 0$. By applying (4.5) and (4.6), we get

$$
P Q^{3} C_{1}(s) f(s, t)+a \kappa^{\prime}(s)=0,
$$


and

$$
Q^{2} f(s, t)\left\{P C_{2}(s)-a\right\}+a \kappa(s)^{2}=0 .
$$

Suppose that $\kappa^{\prime}\left(s_{0}\right) \neq 0$ for some $s_{0}$. Then on an interval $I$, we have $\kappa^{\prime}(s) \neq 0$. On $I, f(s, t)$ is given by

$$
f(s, t)=\frac{-a \kappa^{\prime}(s)}{P Q^{3} C_{1}(s)} .
$$

Hence, by applying $Q=1-\kappa(s) t$, it follows from (4.13) and (4.14) that

$$
a P \kappa(s)^{2} C_{1}(s)-a P \kappa^{\prime}(s) C_{2}(s)+a^{2} \kappa^{\prime}(s)-a P \kappa(s)^{3} C_{1}(s) t=0 .
$$

The coefficient of $t$ in (4.16) must vanish, and hence $C_{1}(s)=0$ on $I$, which contradicts to (4.13). This contradiction shows that $\kappa(s)$ is a constant. Therefore $M$ is a plane or a right circular cone.

Summarizing above, we obtain

Theorem 4.1. Suppose that a GSCS M of polynomial kind has pointwise 1-type Gauss map $G$ of the second kind. Then $M$ is a surface of revolution.

Hence, combining Theorem 4.1 in [5], we get

Corollary 4.2. A GSCS M of polynomial kind has the pointwise 1-type Gauss map $G$ of the second kind if and only if it is a plane or a right circular cone.

\section{REFERENCES}

1. C. Baikoussis \& D. E. Blair: On the Gauss map of ruled surfaces. Glasgow Math. J. 34 (1992), 355-359.

2. C. Baikoussis, B.-Y. Chen \& L. Verstraelen: Ruled surfaces and tubes with finite type Gauss map. Tokyo J. Math. 16 (1993), 341-348.

3. B.-Y. Chen: Total mean curvature and submanifolds of finite type. World Scientific Publ., New Jersey (1984).

4. ___ Finite type submanifolds and generalizations. University of Rome (1985).

5. B.-Y. Chen, M. Choi \& Y.H. Kim: Surfaces of revolution with pointwise 1-type Gauss map. J. Korean Math. Soc. 42 (2005), 447-455.

6. B.-Y. Chen \& P. Piccinni: Submanifolds with finite type Gauss map. Bull. Austral. Math. Soc. 35 (1987), 161-186.

7. U. Dursun: Flat surfaces in the Euclidean space $E^{3}$ with pointwise 1-type Gauss map. Bull. Malays. Math. Sci. Soc.(2) 33 (2010), no. 3, 469-478.

8. D.-S. Kim \& Y.H. Kim: Surfaces with planar lines of curvature. Honam Math. J. 32 (2010), 777-790. 
9. Y.H. Kim \& D.W. Yoon: Ruled surfaces with finite type Gauss map in Minkowski spaces. Soochow J. Math. 26 (2000), 85-96.

10. J. Oprea: Differential geometry and its applications. Prentice Hall, New Jersey, 1997.

Department of Mathematics, Chonnam National University, Kwangue 500-757, Korea

Email address: dosokim@chonnam.ac.kr 